\title{
Worm Climbing the Rubber Rope and Mortgage Loan
}

\author{
Yufa Chen ${ }^{1, \text { a }}$ Yingke Tian $^{1 \mathrm{~b}}$
}

\author{
${ }^{1}$ Zhengzhou Technical College, Basic Teaching Department, Zhengzhou, Henan, 450121 \\ aemail, bemail,
}

Keywords: Integral Model, Differential Equation Model, Comparison, Mortgage Loan

\begin{abstract}
The problem of worm crawled rubber rope, which has studied by some scholars who established the different mathematical models. Compared differential equations model with the integral model and we found that the two models are equivalent. Using this model, different parameters can be calculated for mortgage loans.
\end{abstract}

\section{Introduction}

In the literature [1], the author of the worm crawl rubber rope to promote the problem, and the establishment of an integral model, the worm crawl rubber rope process was described. However, some experts believe that: (1) "In the integral model, the author assumes that the movement of the rubber band in each representative interval has no effect on the movement of the worm and makes it difficult to understand that the integral model is not correct. (2) "the promotion of any problem is moderate, excessive promotion of the problem will lose interest and meaning." I on the above two points and peer experts to discuss.

In addition, the author has further studied the problem, and established a mathematical model from the perspective of differential equations, and compared with the integral model, and then that the two equations are equivalent. Thus, the integral equation is also an accurate model of worm crawling.

In order to compare the study, here to the literature [1] to promote the issue:

The problem has a long and unmate worm from one end of the long rubber rope, with meters / sec (function value,) to the other end of the uniform, while the rubber rope is constantly elongated in the second rubber rope from the rice evenly stretched Long to meters (). So go on, this worm will not climb the other end of the rubber rope?

\section{The Integral Model}

The model of [1] is as follows: Look at whether the worm can climb from the left to the right, that is, look

$$
s=\int_{0}^{n} \frac{a(t)}{f(t)} d t \geq 1
$$

It can be established.

In order to facilitate the discussion later, we discuss two integrators:

$f(t)$ is an Isometric Form.

$$
\begin{aligned}
& \text { If } f(t)=a_{1}+t d,\left(a<a_{1}\right), a(t)=a \text { is constant, then } \\
& \qquad \begin{aligned}
s & =\int_{0}^{n} \frac{a}{f(t)} d t=\int_{0}^{n} \frac{a}{a_{1}+t d} d t \\
& =\left.\frac{a}{d} \ln \left(a_{1}+t d\right)\right|_{0} ^{n}=\frac{a}{d}\left[\ln \left(a_{1}+n d\right)-\ln a_{1}\right] \\
& =\frac{a}{d}[\ln f(n)-\ln f(0)]
\end{aligned}
\end{aligned}
$$


When $\frac{a}{d}[\ln f(n)-\ln f(0)] \geq 1$, the worm climbs from one end of the rubber rope to the other end. $f(t)$ is an Isometric Form.

If $f(t)=a_{1} q^{t},\left(a<a_{1}\right), a(t)=a$ is constant, then

$$
\begin{aligned}
s & =\int_{0}^{n} \frac{a}{f(t)} d t=\int_{0}^{n} \frac{a}{a_{1} q^{t}} d t \\
& =-\left.\frac{a}{a_{1} \ln q} \cdot q^{-t}\right|_{0} ^{n}=\frac{a}{a_{1} \ln q}\left(1-q^{-n}\right)
\end{aligned}
$$

When $\frac{a}{a_{1} \ln q}\left(1-q^{-n}\right) \geq 1$, the worm climbs from one end of the rubber rope to the other end.

\section{Differential Equation Model of Worm Rope}

There is still a discussion of the post-promotion issues.

Figure,

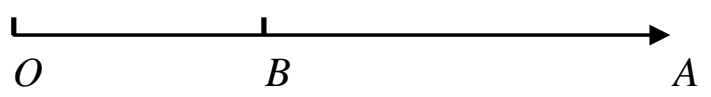

the original length of the rubber rope: $a_{1}=f(0)$, the left end point is $O$, the right end point is $A$, the rubber rope evenly elongated. Assuming at the taim $t$, the speed at $A$ is $b(t)$, as the point $A$ moves, the length of the rubber rope is $f(t)$, the speed at which the worm crawls is $a(t)$, at which point the worm crawls to the point $B$, and the distance from the point $B$ to the point $O$ is $x=x(t)$, that the moving speed of the point $B$ is $\frac{b(t)}{f(t)} x$. then the actual speed of worm crawling is $v=a(t)+\frac{b(t)}{f(t)} x$. But the distance of the worm crawling is $x$, therefore, according to the kinematic meaning of the derivative, we can see $v=x^{\prime}$, thus

$$
x^{\prime}=a(t)+\frac{b(t)}{f(t)} x
$$

This is the differential equation that worms crawl to satisfy. This is a first-order linear differential equation, according to the first-order linear differential equation of the formula (Hou Fengbo, 2003) can be worms crawling equations of motion

$$
x=e^{\int \frac{b(t)}{f(t)} d t}\left[\int a(t) e^{-\int \frac{b(t)}{f(t)} d t} d t+C\right]
$$

The initial condition is: when $t=0, x=0$

In (2), $b(t)$ is the speed of the point $A$ movement, $f(t)$ is the distance from point $A$ to point $O$, and the kinematic meaning of the derivative is known $f^{\prime}(t)=b(t)$, and then

$$
b(t) d t=d f(t)
$$

Substituting (3) into (2)

$$
\begin{gathered}
x(t)=e^{\int \frac{d f(t)}{f(t)}}\left[\int a(t) e^{-\int \frac{d f(t)}{f(t)}} d t+C\right] \\
=e^{\ln f(t)}\left[\int a(t) e^{-\ln f(t)} d t+C\right]
\end{gathered}
$$




$$
=f(t)\left[\int \frac{a(t)}{f(t)} d t+C\right]
$$

Whether the worm from the left end of the rubber rope to climb to the right, that is $x \geq f(t)$, that is to see

$$
\left.\int \frac{a(t)}{f(t)} d t+C \geq 1 \text { (Which } C \text { is determined by " } t=0, \quad x=0 "\right)
$$

Is there any solution? Here, we may wish to set up

$$
g(t)=\int \frac{a(t)}{f(t)} d t+C=\int_{0}^{t} \frac{a(x)}{f(x)} d x
$$

Then $g(t)=\int_{0}^{t} \frac{a(x)}{f(x)} d x$ meet the initial conditions: $t=0, \quad x=0$, Then (5) type into

$$
x(t)=f(t) \int_{0}^{t} \frac{a(x)}{f(x)} d x
$$

(5) into

$$
\int_{0}^{t} \frac{a(x)}{f(x)} d x \geq 1
$$

This conclusion is consistent with (1).

By comparing we see that the above two models are equivalent, the first model is the use of micro-element from the point of view of the integral point of view; the second model is from the perspective of differential issues, the final results of the same way. In contrast, the integral model appears to be more intuitive, concise, and computationally convenient. And the results of the differential equation model are more cumbersome, the process is also more cumbersome.

\section{The Meaning of the Model}

In real life, the repayment of mortgage loans is very similar to this problem. Take the lender, the monthly repayment amount is equivalent to the crawl speed of the worm, the loan interest rate is equivalent to the elongation of the rubber rope. Set $a(t)$ is the monthly repayment amount, the sum of the principal and interest of the bank loan is equivalent to the length of the rubber rope $f(t)$. Calculating the repayment period is equivalent to calculating the time it takes for the worm to climb to the other end of the rubber cord. We discuss in two ways:

Bank Interest Rates Are Calculated on a Single Basis. According to the relevant information, someone loans 10,000 yuan, loans 15 years $=180$ months, the current bank lending rate of 5 years or more is $7.05 \%$, then the monthly interest rate is $\frac{7.05}{1200}=0.5875 \%$, the bank calculated monthly repayment amount is 90.16 yuan. So what is the amount of monthly repayment? At this time, $n=181, \quad f(t)=10,000[1+(t-1) 0.005875]=10000+58.75(t-1)$, it is the difference form, since the monthly repayment amount is fixed, Set it is $A$,

Then, according to 1.1 ,

$$
\begin{gathered}
s=\frac{a}{d}\left\{\ln \left[\left(a_{1}+(n-1) d\right]-\ln a_{1}\right\}\right. \\
=\frac{A}{58.75}\{\ln [10000+58.75 \times 180]-\ln 10000\} \\
=\frac{A}{58.75} \ln \left[1+\frac{58.75 \times 180}{10000}\right] \\
\text { According to } s \geq 1 \text {, we get }
\end{gathered}
$$




$$
\begin{gathered}
\frac{A}{58.75} \ln \left[1+\frac{58.75 \times 180}{10000}\right] \geq 1 \\
A \geq \frac{58.75}{\ln \left[1+\frac{58.75 \times 180}{10000}\right]} \approx 81.69
\end{gathered}
$$

The current interest rate is $4.083333 \%$ o, the loan is 10,000 yuan, the loan is 15 years $=180$ months, The use of similar methods can be provident fund loans monthly repayment amount

$$
A \geq \frac{48.33}{\ln \left[1+\frac{48.33 \times 180}{10000}\right]} \approx 69.63
$$

In turn, we know the amount of money per month, (we only consider the case of a single profit) 1.1 calculation formula, you can calculate how long it takes, but also enough of the bank's principal and interest and the sum. Here, the monthly supply $a=90.16$, bank lending rate of $7.05 \%, d=58.75, a_{1}=10000$

$$
\begin{gathered}
s=\frac{a}{d}\left\{\ln \left[\left(a_{1}+(n-1) d\right]-\ln a_{1}\right\}\right. \\
=\frac{90.16}{58.75}\{\ln [10000+58.75 \times n]-\ln 10000\} \\
=\frac{90.16}{58.75} \cdot \ln \left[1+\frac{58.75}{10000} \times n\right]
\end{gathered}
$$

According to $s \geq 1$, we get

$$
\begin{gathered}
\ln \left[1+\frac{58.75}{10000} \times n\right] \geq \frac{90.16}{58.75} \\
n \geq\left(e^{\frac{58.75}{90.16}}-1\right) \times \frac{10000}{58.75} \approx 155
\end{gathered}
$$

In other words, the monthly repayment of 90.12 yuan, only 12 years and 11 months, about 13 years, (rather than 15 years) can pay off the bank's loans.

The results here and the bank to implement the actual standard deviation is more, mainly because the bank has its own set of algorithms. The bank's real interest rate is much larger than 7.05\%. So what is the real interest rate of the bank? We can also use the above model to calculate:

Here $a=90.12, n=181, a_{1}=10000, d$ is unknown, according to the 2.1 model

$$
\begin{gathered}
s=\frac{a}{d}\left\{\ln \left[\left(a_{1}+(n-1) d\right]-\ln a_{1}\right\}\right. \\
=\frac{90.12}{d}\{\ln [10000+d \times 180]-\ln 10000\} \\
=\frac{90.12}{d} \ln \left[1+\frac{180 d}{10000}\right]
\end{gathered}
$$

According to $s \geq 1$, we get

$$
\begin{gathered}
\frac{90.12}{d} \ln \left[1+\frac{180 d}{10000}\right] \geq 1 \\
d \geq \frac{10000}{180}\left(e^{\frac{d}{90.12}}-1\right)
\end{gathered}
$$

After calculation, the bank's real interest rate is about $13.53 \%$.

It should be noted that the bank's calculation method is discrete, that is, monthly interest. In fact, the discrete algorithm and the continuous algorithm is very small, there is no comparison. 
Bank Interest Rate Calculated by Compound Interest. At this time, $n=181$, $f(t)=10,000\left(\frac{1005.875}{1000}\right)^{t-1}=10,000(1.005875)^{t-1}$ repayment amount is fixed,

Then, according to 1.2,

$$
\begin{gathered}
s=\frac{a}{a_{1} \ln q}\left(1-q^{1-n}\right)=\frac{A}{10,000 \cdot \ln (1.005875)}\left(1-1.005875^{-180}\right) \\
\text { According to } s \geq 1 \text {, we get } \\
\frac{A}{10,000 \cdot \ln (1.005875)}\left[1-(1.005875)^{-180}\right] \geq 1 \\
A \geq \frac{10,000 \cdot \ln (1.005875) \cdot(1.005875)^{180}}{(1.005875)^{180}-1} \approx 91.25
\end{gathered}
$$

Provident fund loans, the use of the above model, the corresponding data into available,

$$
A \geq \frac{10,000 \cdot \ln (1.0048333) \cdot(1.0048333)^{180}}{(1.0048333)^{180}-1} \approx 80.02
$$

This result is not the same as the bank's calculations. In fact, if the compound interest is calculated, the borrower needs to pay more interest.

We can also use the 1.2 model to calculate: according to the monthly and bank interest rates to calculate the repayment period, or according to the repayment period and month to calculate the actual interest rate. This is not repeated here.

\section{References}

[1] Chen Yufa .2012. Mathematical model of worm crawling rubber rope[J]. Journal of Beijing Institute of Education (4): 11-14.

[2] Hou Fengbo .2003. Higher Mathematics[M]. Beijing: Higher Education Press, 145. 